\title{
CORRESPONDENCE
}

\section{An unusual cause of haemoptysis in a patient with asthma}

\section{To the Editor:}

A 35-yr-old Asian nonsmoking housewife was seen in the asthma clinic in September 2006 with cough and haemoptysis. Her past history included atopic brittle asthma [1] since childhood with two intensive care unit (ICU) admissions in the past requiring mechanical ventilation, the latest being 1 month previously.

She was on maximal treatment for her asthma, and had recently been admitted to the ICU in June-August 2006, initially with an exacerbation of asthma with bilateral pneumothoraces, subsequently complicated by sepsis and acute respiratory distress syndrome. She was slow to wean from the ventilator and required a mini-tracheostomy for 19 days. In the period following discharge, she had been reviewed weekly in the clinic. She complained of a dry cough and dyspepsia, which was treated with a proton pump inhibitor and a prokinetic. Due to persistence of symptoms, she was also given a trial of prednisolone $30 \mathrm{mg}$ daily for 5 days.

At the clinic appointment in September 2006, her cough had become productive of yellow sputum with streaks of blood. She also complained of a nonpleuritic left-sided chest pain around the site of the previous chest drain. On examination, there was widespread bilateral wheeze and crepitations in the right base with a very tender left chest wall. She was admitted for treatment of acute exacerbation of asthma and further investigation.

Her haemoglobin remained stable at $13.3 \mathrm{~g} \cdot \mathrm{dL}^{-1}$ and inflammatory markers were normal. Blood gases showed a mild hypoxia (arterial oxygen tension $9.12 \mathrm{kPa}$ on room air). Chest radiograph demonstrated fibrotic upper lobe changes, which were long standing. A computed tomography pulmonary angiogram performed the next day excluded pulmonary embolus, and a bronchoscopy was arranged for the following day.

The bronchoscopy revealed an unexpected finding. A synthetic white object was found embedded in the upper trachea (fig. 1a) extending into the right main bronchus (fig. 1b). The patient was referred urgently to the ear, nose and throat (ENT) surgeons for assistance in removal of the device, thought to be part of the previous tracheostomy tube.

Thoracic computed tomography was arranged, specifically to examine the trachea. A review of her recent ICU admission documentation confirmed an uneventful percutaneous tracheostomy insertion.

The ENT surgeon tried to remove the foreign body via rigid bronchoscopy, but was unsuccessful; therefore, surgical removal was attempted. The old mini-tracheostomy wound was opened through an incision made above the second tracheal ring, and the foreign body identified approximately $0.7 \mathrm{~cm}$ below this. Therefore, the trachea was opened further and it became obvious that the Luer lock fitting of the minitracheostomy introducer was embedded in the tracheal musculature (fig. 1a). This was removed (fig. 1c) and both incisions were repaired. The haemoptysis and dyspnoea subsequently settled, and the patient recovered.

Our patient presented with worsening symptoms of asthma, unresponsive to standard therapy [2], associated with haemoptysis. The differential diagnosis of haemoptysis in a brittle asthmatic includes: allergic bronchopulmonary aspergillosis [3], pulmonary embolus and bronchiectasis. Less likely causes include neoplasm, Churg-Strauss syndrome [4], tuberculosis, parasitic infections [5] or a foreign body.

In this case, the mini-tracheostomy introducer had not been retrieved and became embedded in the trachea. The procedure and protocol for percutaneous tracheostomy at our institution has since been reviewed, and amended, in order to prevent a recurrence of this problem.

This clinical case demonstrates how subtle details in a patient's past medical history may provide clues to the current presentation and highlights the importance of a good clinical history. The possibility of an iatrogenic aetiology for an
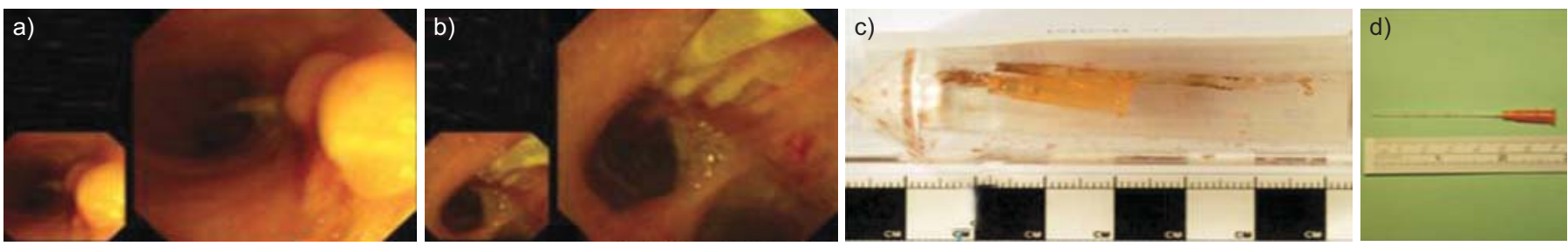

FIGURE 1. Bronchoscopy findings: a) Luer lock embedded in tracheal musculature (at the level of the trachea); and b) mini-tracheostomy introducer entering right main bronchus (at the level of the carina). c) The mini-tracheostomy introducer removed from the patient; and d) a sample mini-tracheostomy introducer 
unexplained new symptom should always be borne in mind, particularly after a period of prolonged hospitalisation.

\section{Irfan, D. Gnananandha and M. Munavvar}

Lancashire Teaching Hospitals, Preston, UK.

Correspondence: M. Irfan, Respiratory Medicine, Royal Preston Hospital, Preston, PR2 9HT, UK. E-mail: mujammil76@ doctors.org.uk

Statement of Interest: None declared.

Provenance: Submitted article, peer reviewed.

\section{REFERENCES}

1 Barnes PJ, Woolcock AJ. Difficult asthma. Eur Respir J 1998; 12: 1209_ 1218.

2 Robinson DS, Campbell DA, Durham SR, et al. Systematic assessment of difficult-to-treat asthma. Eur Respir J 2003; 22: 478-483.

3 Shah A, Panjabi C. Contemporaneous occurrence of allergic bronchopulmonary aspergillosis, allergic aspergillus sinusitis and aspergilloma. Ann Allergy Asthma Immunol 2006; 96: 874-878.

4 Manganelli $\mathrm{P}$, Fietta $\mathrm{P}$, Carotti M, et al. Respiratory system involvement in systemic vasculitides. Clin Exp Rheumatol 2006; 24: Suppl. 41, S48-S59.

5 Ochoa MD, Ramirez-Mendoza P, Ochoa G, et al. Bronchial nodules produced by Strongyloides stercoralis as the cause of bronchial obstruction. Arch Bronchoneumol 2003; 39: 524-526.

DOI: $10.1183 / 09059180.00003009$ 